\title{
IMPLEMENTACIÓN DE UN SISTEMA DE MONITOREO DE ÁREA EXTENDIDA WAMS EN EL Sistema Nacional Interconectado DEL ECUADOR SNI
}

\author{
Aharon De La Torre ${ }^{1, *}$, Jaime Cepeda ${ }^{2}$ y J. C. Herrera ${ }^{3}$
}

\section{Resumen}

En el presente trabajo se describen los principales componentes del sistema WAMS y la medición fasorial en sistemas eléctricos; se refieren criterios para la ubicación de PMU y elementos asociados en el sistema eléctrico. Posteriormente se hace una descripción del sistema WAMS instalado en el sistema eléctrico de Ecuador y se describen las características funcionales de la aplicación (software) de WAMS disponible en el Centro Nacional de Control de Energía - CENACE. Seguidamente se aplican los conceptos de ingeniería en el análisis de la información obtenida con el sistema WAMS instalado, presentándose los primeros resultados. Finalmente, y con base a los análisis realizados, se establecen los principales elementos de diseño y estrategias para la implementación de un sistema WAMS generalizado en el Sistema Nacional Interconectado - SNI del Ecuador.

Palabras clave: Concentrador de datos fasoriales, estabilidad oscilatoria, fasor, modos de oscilación, sincrofasor, sistema de monitoreo de área extendida, unidad de medición fasorial.

\section{Abstract}

This paper describes the WAMS architecture and its main components, and depicts some criteria for the location of the elements of WAMS. Subsequently, a description of the application (software) of WAMS available at Centro Nacional de Control de EnergíaCENACE, is carried out. A technical analysis of the information obtained from the installed WAMS, as well as a presentation of the first results of the implementation of WAMS in the SNI of Ecuador, are also deployed in this document. Finally, based on the analysis performed, major design elements and strategies for implementation of a generalized WAMS in the SNI of Ecuador are identified.

Keywords: Oscillatory stability, oscillation modes, phasor, phasor data concentrator synchrophasor, phasor measurement unit, wide area monitoring system.

\footnotetext{
$\overline{1, *}$ Graduado en Ingeniería Eléctrica, Universidad Politécnica Salesiana, sede Quito - Campus Kennedy. Autor para correspondencia : adltorre@cenace.org.ec

${ }^{2}$ Jefe del Área de Investigación y Desarrollo de la Corporación CENACE. Autor para correspondencia ${ }^{\circ}$ jcepeda@ cenace.org.ec

${ }^{3}$ Docente de Ingeniería Eléctrica, Universidad Politécnica Salesiana, sede Quito - Campus Kennedy. Autor para correspondencia jherrera@cenace.org.ec
}

Recibido: 04-11-2013, Aprobado tras revisión: 27-11-2013.

Forma sugerida de citación: De La Torre, A.; Cepeda, J. y Herrera, J. (2013). "Implementación de un sistema de monitoreo de área extendida WAMS en el Sistema Nacional Interconectado del Ecuador SNI". INGENIUS. N. ${ }^{\circ} 10$, (Julio-Diciembre). pp. 34-43. ISSN: 1390-650X. 


\section{Introducción}

El estado del arte de la planificación, operación y análisis posoperativo de los Sistemas Eléctricos de Potencia exige la utilización de nuevas herramientas que permitan extender la filosofía de operación utilizada en una Subestación Eléctrica del Sistema de Transmisión a un Sistema Eléctrico de Potencia - SEP distribuido geográficamente, que por lo general se encuentra constituido por varias subestaciones de carga, centrales de generación e interconexiones con otras áreas.

Esta nueva concepción en el proceso de gestión de sistemas eléctricos de potencia se denomina WAMS (Wide Area Measurement System) e implica la utilización de una combinación de tecnologías de medición fasorial, sistemas de comunicaciones y aplicaciones que proporcionan observación dinámica del sistema de potencia con alta precisión en las mediciones. Estas mediciones son sincronizadas con la señal de tiempo de un Sistema Global de Posicionamiento (GPS) y los tiempos de adquisición de datos están en el orden de milisegundos [1], lo que proporciona a los ingenieros de operación, planificación y análisis posoperativo, mejores herramientas de supervisión y análisis del sistema de potencia.

\section{Principales componentes del sistema WAMS}

\subsection{Unidad de medición fasorial (PMU)}

Las PMU (Phasor Measurement Unit) son dispositivos que permiten estimar sincrofasores (fasores que tienen como referencia angular a una onda coseno de frecuencia nominal, sincronizada con el tiempo UTC Universal Time Coordinated) de las ondas sinusoidales de corriente y voltaje AC, en diferentes nodos de un SEP [2]. Para calcular un sincrofasor la PMU utiliza un algoritmo de estimación fasorial. Estos algoritmos utilizan un número $N$ de muestras en el tiempo para efectuar la estimación del fasor. El algoritmo más comúnmente utilizado es la transformada discreta de Fourier [2]. La alta precisión, velocidad de respuesta y sincronización de tiempo hacen de las PMU equipos apropiados para el monitoreo global en estado estable y dinámico, así como para aplicaciones en protección y control, como parte básica de un sistema de monitorio de área extendida [3].

\subsection{Concentrador de datos de fasores (PDC)}

La función del PDC (Phasor Data Concentrator) es recibir y almacenar la información de los datos medidos por las PMU y dependiendo de la complejidad del sistema de otros PDC. En resumen las funciones que realiza un PDC son [4]:
- Organizar los datos fasoriales de acuerdo a la estampa de tiempo, concentrando todas las medidas de la red de PMU.

- Enviar información a los históricos o hacia otros PDC.

- Registrar las pérdidas de conexión y de recepción de información.

- Registrar los errores de información e insertar banderas que indican la calidad de los datos.

Los PDC de acuerdo a su utilización y ubicación pueden ser locales, regionales y centrales o Súper PDC, dependiendo de la arquitectura utilizada. Y pueden ser de hardware o de software.

Para el caso del CENACE los PDC utilizados son de software y están instalados en el mismo servidor en donde se encuentran las aplicaciones WAMS. Estos PDC son del tipo Central o Super PDC ya que concentran la información de todas las PMU instaladas en el SNI.

\subsection{Estándar IEEE C37.118}

Este estándar se aplica a las mediciones sincrofasoriales utilizadas por los sistemas WAMS en condiciones normales de operación, ver [5].

\section{Ubicación de PMUs en el SNI}

Uno de los criterios para la ubicación de las PMU, es la observabilidad, maximizando la redundancia pero empleando la menor cantidad de PMU, sin que esto signifique la pérdida de observabilidad global del sistema. Varios autores han desarrollado modelos matemáticos que resuelven el problema de la ubicación óptima de PMU, en un sistema eléctrico, utilizando métodos de optimización, un ejemplo de estas propuestas se encuentra en [6].

Otro criterio es el de dar prioridad a las barras más relevantes del sistema eléctrico para la instalación de las PMU, ya que de acuerdo a la barra y su ubicación los desempeños son distintos, pudiéndose llegar a obtener mucha información de interés, por ejemplo datos asociados a las barras centrales o principales, interconexiones, barras de alta generación o de carga, entre otras.

En principio, la ubicación de las PMU en el SNI se resolverá de acuerdo a lo que se necesite monitorear, tomando el criterio de barras relevantes, y aprovechando la experiencia operativa. La aplicación de estos criterios definidos por el grupo del proyecto WAMS, se presentarán más adelante.

Una vez implantado el sistema WAMS inicial y habiéndose obtenido los primeros resultados del análisis de la información de los eventos registrados en 
el SNI, se podrán identificar nuevas zonas para la instalación de PMU, valorar la plausibilidad de su reubicación o establecer un cronograma estratégico de dotación de nuevo equipamiento, todo esto con el fin de alcanzar en el futuro cercano la observabilidad absoluta del sistema.

\subsection{Ubicación considerando la supervisión angular del SEP}

Como estrategia inicial, las PMU se colocan principalmente en las barras del anillo troncal del sistema de transmisión del SEP, en las subestaciones frontera de interconexiones eléctricas y en barras de los grandes centros de carga del SEP.

\subsection{Ubicación considerando la estabilidad oscilatoria del SEP}

La ubicación de las PMU está ligada a los estudios eléctricos orientados a identificar subestaciones susceptibles a presentar oscilaciones, complementándose con el criterio de colocarlas en los nodos asociados a las interconexiones, con el fin de obtener información de posibles oscilaciones interárea.

\subsection{Sintonización de parámetros eléctricos de elementos del SEP}

Para el caso de líneas de transmisión, las PMU se ubican en las barras que conectan con la línea de transmisión cuyos parámetros serán analizados.

\subsection{Calibración de parámetros en PSS (Power System Stability), reguladores de veloci- dad y AVRs (Automatic Voltaje Regula- tors)}

Las PMU se ubican en la barra de bornes del generador. Para el caso de transformadores, se ubicarán las barras de alto y bajo voltaje.

\subsection{Subestaciones críticas en la operación del SEP}

Es necesario también determinar los elementos que son críticos en el sistema y que necesitan una mejor supervisión. Este caso comprende líneas de transmisión y transformadores con altas transferencias de potencia, cuyo disparo involucra riesgo en la operación del SEP.

\section{Descripción de la aplicación WAMS del CENACE}

Los sistemas de medición sincrofasorial utilizando PMU han creado un nuevo entorno en la operación de SEP dando lugar a la creación de una clase de aplicaciones que están basadas en mediciones y no dependen de ningún modelo matemático del sistema eléctrico. Estas aplicaciones están en pleno desarrollo y todavía no se dispone de un conjunto definido de aplicaciones estándares, por lo que cada empresa desarrolladora dispone de sus propias aplicaciones WAMS con sus características determinadas.

La aplicación WAMS disponible en CENACE provee la funcionalidad de PDC, que además de gestionar las PMU del sistema, contiene una serie de funciones, módulos de cálculo, drivers y herramientas para análisis gestionables a través de una interface de usuario personalizable y que permite al operador tener una consciencia situacional del SEP.

\subsection{Características de interface humano - máquina [7]}

- Provee información histórica y en tiempo real de las principales variables del sistema.

- Incluye información jerárquica multiusuario.

- Permite la visualización de información en tiempo real de todas las variables disponibles mediante gráficos polares, gráficos en función del tiempo, gráficos 3D o diagramas tabulares.

- Presenta oscilogramas que permiten almacenar en gráficos datos de eventos del sistema para análisis.

- Presenta el contorneo de diferencias angulares del SEP.

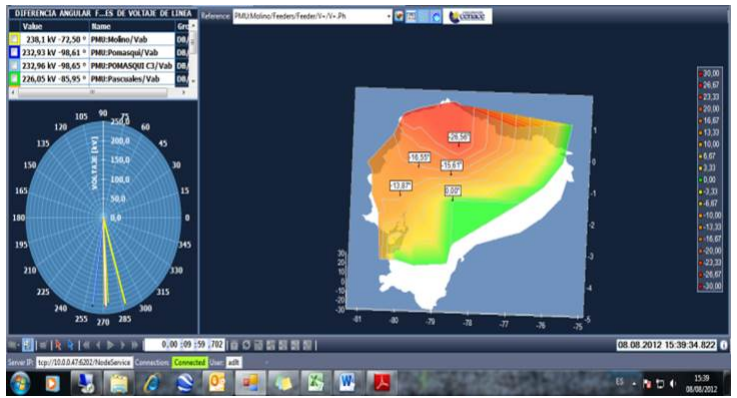

Figura 1. Despliegue personalizado de diferencias angulares. Fuente: CENACE, Aplicación WAProtector.

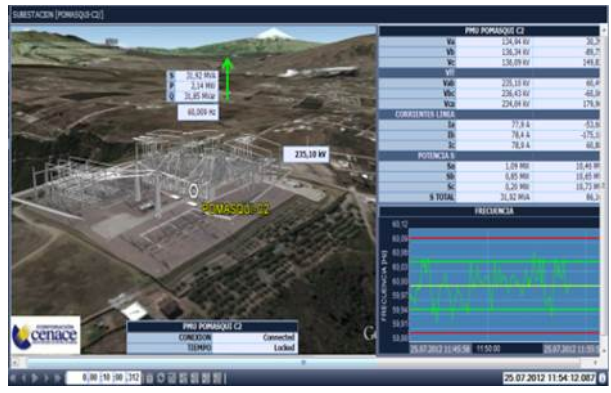

Figura 2. Despliegue de subestación. Fuente: CENACE, Aplicación WAProtector. 


\subsection{Características funcionales [7]}

- Aplicación Central PDC (gestión de PDC).

- Almacenamiento para datos de PMU y aplicaciones WAMS.

- Administración de notificaciones de alarmas y eventos.

- Integración con sistemas SCADA/EMS.

- Administración de estabilidad oscilatoria.

- Detección y resincronización de islas.

- Estabilidad de voltaje en líneas de transmisión.

- Análisis de calidad de energía.

\section{Presentación de primeros análisis y resultados}

La aplicación WAProtector de ELPROS, para la gestión del sistema WAMS posee un algoritmo propietario que entrega resultados de la composición modal de señales de potencia activa. Estos datos requieren de un adecuado procesamiento con el objetivo de obtener información valiosa sobre el comportamiento oscilatorio del sistema que permita caracterizarlo adecuadamente.

La caracterización oscilatoria del SNI, será la referencia para la estructuración de acciones de control preventivo (despacho programado de generación o calibración de PSS), así como acciones de control correctivo (re-despacho de unidades de respuesta rápida o modificaciones topológicas), que en su conjunto permitirán mejorar la seguridad operativa del SEP.

Este trabajo presenta una propuesta metodológica que permite caracterizar el comportamiento oscilatorio del SNI, basada principalmente en el análisis estadístico de los modos oscilatorios pobremente amortiguados.

\subsection{Estabilidad oscilatoria}

Al producirse variaciones de carga o generación en sistemas de potencia, los generadores tratan de encontrar nuevos estados de operación estable, produciéndose oscilaciones de potencia y frecuencia ocasionadas por los intercambios de energía eléctrica entre dichas unidades. Las oscilaciones son caracterizadas mediante los denominados modos de oscilación que pueden clasificarse, en forma general, en los siguientes [8]:

Los modos locales se asocian con oscilaciones entre rotores de un grupo de generadores cercanos unos a otros. Estas oscilaciones presentan frecuencias en el rango de 0,7 a $2,0 \mathrm{~Hz}$. Los modos interárea, por el contrario, son causados por interacciones entre grandes grupos de generadores oscilando entre ellos. Estos modos presentan rangos de frecuencia entre $0,1 \mathrm{a} 0,7 \mathrm{~Hz}$.

Existen dos tipos de modos oscilatorios adicionales, causados por controladores de diferentes tipos de componentes (llamados modos de control), o por oscilaciones que ocurren en los sistemas rotacionales de los sistemas turbina-generador de las unidades generadoras (denominados modos torsionales).

Este tipo de oscilaciones presentan un rango de frecuencias asociadas bastante amplio. Dentro de los modos de control existen unos de muy baja frecuencia $(0,01 \mathrm{~Hz}-0,1 \mathrm{~Hz})$ que aparecen en sistemas con alta penetración hidráulica y que están asociados a la inadecuada sintonización de los controladores de velocidad de las unidades de generación hidráulicas. Este tipo de modo de control se ha detectado en ocasiones en el sistema ecuatoriano, en los análisis del Área de Análisis de la Operación del CENACE.

El análisis modal [8] es la herramienta más utilizada para analizar las oscilaciones en un SEP. Consiste en la determinación de los modos de oscilación y el análisis de sus correspondientes frecuencias modales complejas (es decir, de los valores propios de cada modo: $\sigma_{i} \pm \mathrm{j} \omega_{i}$ ). La estabilidad oscilatoria se cumple cuando todos los modos presentan un amortiguamiento positivo. La frecuencia y el amortiguamiento de la oscilación pueden determinarse a partir del valor propio de la siguiente manera:

$$
\begin{gathered}
f_{i}=\frac{\omega_{i}}{2 \pi} \\
\zeta_{i}=\frac{-\sigma_{i}}{\sqrt{\sigma_{i}^{2}+\omega_{i}^{2}}}
\end{gathered}
$$

donde $f_{i}$ y $\zeta_{i}$ son la frecuencia de oscilación y la razón de amortiguamiento del $i$-ésimo modo, respectivamente. El análisis modal se basa en la modelación matemática completa del SEP, lo que involucra altos tiempos de cómputo para grandes sistemas. Esto constituye una limitación para su aplicación en tiempo real [9]. Con el objetivo de superar esta limitante, se han estructurado diferentes propuestas para evaluar las oscilaciones en tiempo real.

Un enfoque utiliza modelos y herramientas computacionales inteligentes (como redes neuronales artificiales) para estimar el riesgo oscilatorio del SEP [10], mientras que una alternativa, con mayor robustez por sus facilidades de aplicación propone el uso de mediciones sincrofasoriales y su consiguiente procesamiento a través de algoritmos de identificación modal [9]. 


\subsection{Evaluación de oscilaciones}

El sistema instalado permite monitorear en tiempo real la composición modal de las señales de potencia activa registradas por cada una de las PMU, a través de la aplicación de un algoritmo propietario de estimación modal. Este monitoreo proporciona señales de alerta temprana al operador y le permiten visualizar la presencia de condiciones de vulnerabilidad del sistema eléctrico a causa de la inestabilidad oscilatoria.

Adicionalmente WAProtector permite recuperar información de la frecuencia, el amortiguamiento y la amplitud de los modos oscilatorios en cada una de las PMU. Esta información se procesa mediante herramientas que recopilan y organizan los datos por cada PMU y en cada hora.

Con el objetivo de realizar un análisis sistémico de los modos oscilatorios en cada hora, este debe contemplar a todas las áreas coherentes del sistema (puesto que en cada área coherente pueden presentarse modos particulares). Por tanto, es necesario analizar la información oscilatoria en todas las PMU que se disponen en el sistema, en primera instancia; o en su defecto, determinar aquellas PMU que brindan información redundante (es decir que están ubicadas en la misma área coherente) y analizar únicamente las que permiten observar modos diferentes. Esta información permitirá evaluar adecuadamente el comportamiento oscilatorio global del sistema.

Del total de la información, se analizan únicamente los modos que presenten un amortiguamiento inferior al límite que establezca el estudio correspondiente. Sobre la base de este criterio, se ha usado como referencia aquellos modos que presentan valores inferiores al 5\% (límite de alerta) y al 3\% (límite de alarma). Así, se identifican y se resumen los modos cuyo amortiguamiento es inferior $3 \%$ o $5 \%$, la hora en la que ocurrieron estas oscilaciones y la PMU que permitió su observación.

De estos modos, se resaltan, como referencia, aquellos cuyas amplitudes son de más de $1 \mathrm{MW}$ (los valores de amplitud correspondientes para cada modo de oscilación se determinan posteriormente a través de un análisis estadístico, como se presenta más adelante). Es posible ahora identificar, según el rango de frecuencias, los modos poco amortiguados que mayormente se presentan, dónde son observables y en qué periodos de demanda se presentan como dominantes.

Este análisis permite identificar aquellos modos que evidencian amortiguamientos bajos e incluso negativos, y que podría eventualmente conllevar a problemas de estabilidad del sistema, además de determinar la influencia en la excitación de estos modos de ciertas centrales hidráulicas, térmicas y estados operativos.
Una vez determinados los modos oscilatorios más críticos, se procede a realizar un análisis más detallado de estos, en las horas en las que las oscilaciones hayan sido determinadas. Por tanto, se requiere una búsqueda más exhaustiva, usando las aplicaciones gráficas del software tal que permita encontrar un evento que dispare una oscilación, incluso cuando esta oscilación no sea de magnitud considerable. Resulta apropiado, además, determinar las centrales cuya desconexión dispara cierto tipo de oscilaciones en el sistema.

\subsection{Análisis de estabilidad oscilatoria para la semana del 12 al 18 de agosto de 2013}

Se han realizado análisis semanales de estabilidad oscilatoria con la información del sistema WAMS, desde mayo del 2013 hasta la fecha, con propósitos ilustrativos se presenta el análisis estadístico de la información correspondiente al período del 12 al 18 de agosto de 2013. Del total de información, se analizan únicamente los modos que presentan un amortiguamiento horario promedio inferior al 5\%, el cual se considera como el límite permitido de amortiguamiento para las oscilaciones que se presentan en el Sistema Nacional Interconectado.

Dentro de estos modos poco amortiguados se han determinado modos de control $(0.01-0.1 \mathrm{~Hz}$ y mayores a $4 \mathrm{~Hz})$, modos interárea $(0.1-0.7 \mathrm{~Hz})$ y modos locales $(0.7 \mathrm{~Hz}-2.0 \mathrm{~Hz})$.

\subsubsection{Modos de control de muy baja frecuencia}

En el período de análisis, se han determinado modos de control poco amortiguados, ubicados en el rango de frecuencias entre $0.011 \mathrm{~Hz}$ y $0.099 \mathrm{~Hz}$. Cabe destacar la presencia de diez modos que presentaron amortiguamientos negativos (resaltados con contorno rojo en la Figura 3), indicando posibles riesgos de estabilidad oscilatoria en el sistema.

Adicionalmente, se realiza un análisis estadístico de las amplitudes que alcanzaron los modos de control poco amortiguados durante el período de análisis. Los resultados arrojan que en el $48.54 \%$ de los casos la amplitud es mayor a $2.84 \mathrm{MW}$ y que la mayor amplitud fue de 6.84 MW.

La Figura 4, presenta el histograma de las amplitudes correspondientes a los modos de control en el período de análisis.

La Tabla 1, presenta un resumen de los modos cuyo amortiguamiento fue inferior al valor crítico de $3 \%$, la fecha y hora en la que ocurrieron estas oscilaciones, así como la PMU que permitió su observación. Es factible apreciar que estos modos de control se presentan especialmente en horas de demanda media, pero están presentes también en demanda máxima y mínima. 


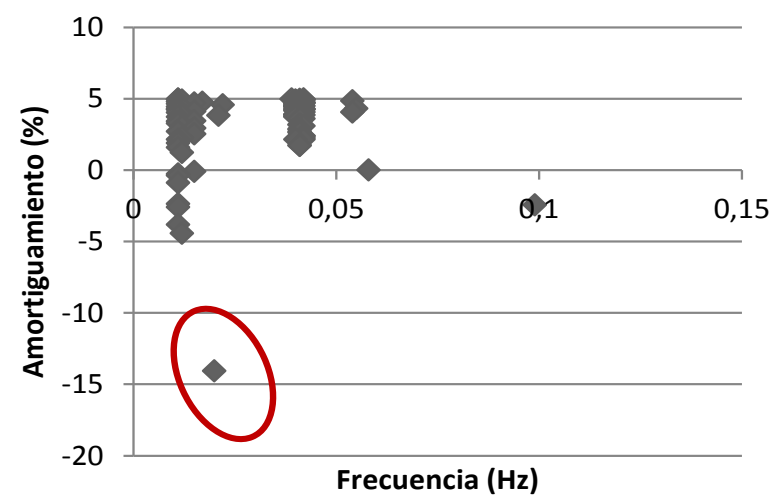

Figura 3. Modos de control con amortiguamiento inferior al $5 \%$.

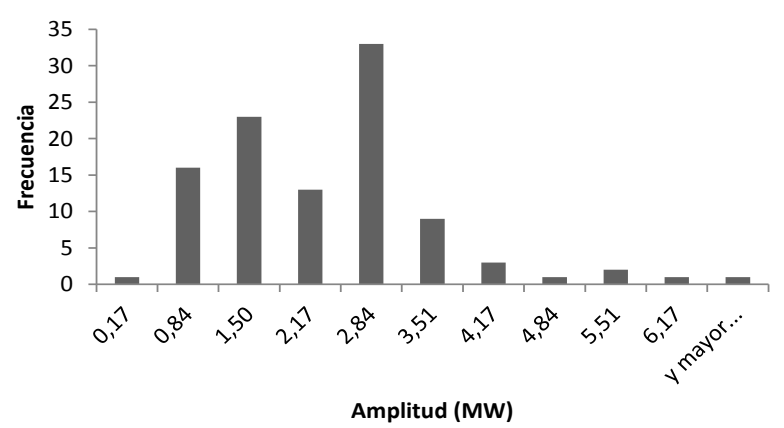

Figura 4. Histograma de amplitudes de los modos de control poco amortiguados.

Tabla 1. Modos de control con amortiguamiento inferior al $3 \%$.

\begin{tabular}{lccccc}
\hline & & & \multicolumn{3}{c}{ Modo oscilatorio } \\
\cline { 4 - 6 } PMU & Hora & Fecha & $\begin{array}{c}\text { Frec. } \\
\text { (Hz) }\end{array}$ & $\begin{array}{c}\text { Amp. } \\
\text { (MW) }\end{array}$ & $\begin{array}{c}\text { Amor. } \\
\text { (\%) }\end{array}$ \\
\hline SROS_TOTO2_230 & $10: 00$ & $15 / 08 / 2013$ & 0.011 & 1.32 & 1.89 \\
MOLI_AT1_138 & $12: 00$ & $15 / 08 / 2013$ & 0.041 & 4.52 & 1.75 \\
MOLI_TOTO_230 & $10: 00$ & $13 / 08 / 2013$ & 0.041 & 2.72 & 1.71 \\
MOLI_TOTO_230 & $18: 00$ & $15 / 08 / 2013$ & 0.011 & 1.76 & 1.6 \\
ZHOR_MILA2_230 & $3: 00$ & $14 / 08 / 2013$ & 0.012 & 0.54 & 1.22 \\
SROS_SDOM1_230 & $19: 00$ & $18 / 08 / 2013$ & 0.015 & 2.46 & -0.1 \\
MOLI_AT1_138 & $18: 00$ & $15 / 08 / 2013$ & 0.011 & 2.21 & -0.26 \\
MOLI_PASC1_230 & $23: 00$ & $14 / 08 / 2013$ & 0.011 & 0.72 & -0.38 \\
MOLI_AT1_138 & $10: 00$ & $14 / 08 / 2013$ & 0.011 & 2.24 & -0.89 \\
PASC_MOLI2_230 & $18: 00$ & $16 / 08 / 2013$ & 0.011 & 0.49 & -2.36 \\
MOLI_TOTO_230 & $3: 00$ & $12 / 08 / 2013$ & 0.099 & 0.51 & -2.45 \\
MOLI_PASC1_230 & $18: 00$ & $16 / 08 / 2013$ & 0.011 & 0.52 & -2.58 \\
PASC_MOLI_230 & $18: 00$ & $16 / 08 / 2013$ & 0.011 & 0.39 & -3.81 \\
QUEV_ATT_138 & $19: 00$ & $12 / 08 / 2013$ & 0.012 & 0.17 & -4.43 \\
SROS_POMA1_230 & $10: 00$ & $16 / 08 / 2013$ & 0.02 & 1.48 & -14.06 \\
\hline
\end{tabular}

\subsubsection{Modos de control de alta frecuencia}

En la Figura 5, se observan modos oscilatorios en el rango de frecuencia $5.79 \mathrm{~Hz}-5.87 \mathrm{~Hz}$, particularmente en la zona de Quevedo, se estima que estos modos están asociados con modos de control producidos por la generación de la zona, lo cual se encuentra bajo análisis.

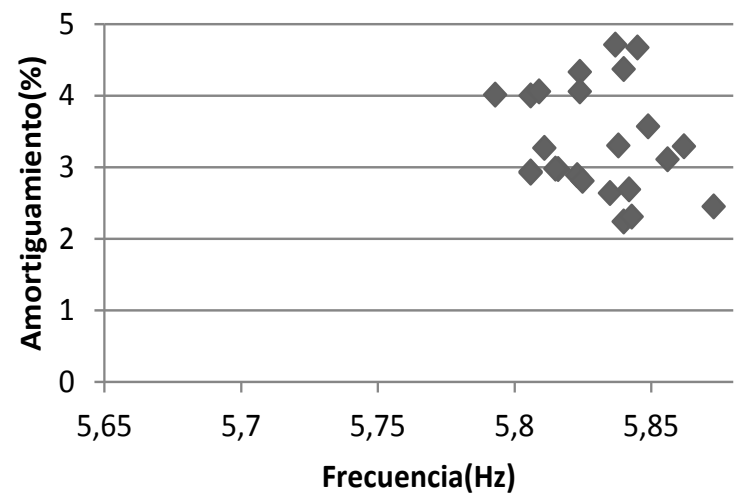

Figura 5. Modos de control de alta frecuencia con amortiguamiento inferior al $5 \%$.

La Figura 6, presenta el histograma obtenido del análisis estadístico de las amplitudes que alcanzaron los modos de control de alta frecuencia poco amortiguados durante el período de análisis. De los resultados se observa que en el $87.5 \%$ de los casos, la amplitud es mayor a $0.25 \mathrm{MW}$ y que la mayor amplitud fue de $0.35 \mathrm{MW}$.

La Tabla 2, presenta un resumen de los modos cuyo amortiguamiento fue inferior al valor de $3 \%$. Es factible apreciar que estos modo de control de alta frecuencia, se presentan especialmente en horas de demanda media, y son observables solamente en la zona de Quevedo.

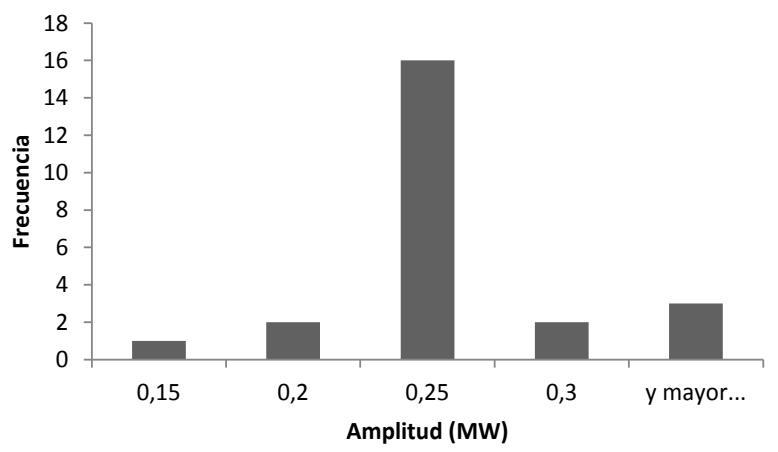

Figura 6. Modos de control de alta frecuencia con amortiguamiento inferior al $5 \%$.

Tabla 2. Modos de control de "alta frecuencia" con amortiguamiento inferior al $3 \%$.

\begin{tabular}{cccccc}
\hline & & & \multicolumn{3}{c}{ Modo oscilatorio } \\
\cline { 4 - 6 } PMU & Hora & Fecha & $\begin{array}{c}\text { Frec. } \\
\text { Pmp. }\end{array}$ & $\begin{array}{c}\text { Ampr } \\
\text { (MW) }\end{array}$ & $\begin{array}{c}\text { Amor } \\
\text { (\%) }\end{array}$ \\
\hline QUEV_ATT_138 & $11: 00$ & $14 / 08 / 2013$ & 5.815 & 0.23 & 2.98 \\
QUEV_ATT_138 & $12: 00$ & $14 / 08 / 2013$ & 5.816 & 0.23 & 2.97 \\
QUEV_ATT_138 & $9: 00$ & $14 / 08 / 2013$ & 5.806 & 0.21 & 2.94 \\
QUEV_ATT_138 & $13: 00$ & $13 / 08 / 2013$ & 5.806 & 0.2 & 2.92 \\
QUEV_ATT_138 & $15: 00$ & $14 / 08 / 2013$ & 5.823 & 0.25 & 2.89 \\
QUEV_ATT_138 & $15: 00$ & $13 / 08 / 2013$ & 5.825 & 0.22 & 2.81 \\
QUEV_PASC1_230 & $12: 00$ & $13 / 08 / 2013$ & 5.842 & 0.35 & 2.69 \\
QUEV_ATT_138 & $14: 00$ & $14 / 08 / 2013$ & 5.835 & 0.25 & 2.64 \\
QUEV_ATT_138 & $9: 00$ & $13 / 08 / 2013$ & 5.873 & 0.24 & 2.45 \\
QUEV_ATT_138 & $13: 00$ & $14 / 08 / 2013$ & 5.843 & 0.24 & 2.31 \\
QUEV_ATT_138 & $10: 00$ & $13 / 08 / 2013$ & 5.84 & 0.25 & 2.24 \\
\hline
\end{tabular}




\subsubsection{Modos interárea}

Se determina la existencia de modos interárea poco amortiguados en el rango de frecuencia de $0.42 \mathrm{~Hz}-$ $0.62 \mathrm{~Hz}$, como se observa en la Figura 7.

La Figura 8, presenta el histograma obtenido del análisis estadístico de las amplitudes que alcanzaron los modos interárea poco amortiguados durante el período de análisis. De los resultados se observa que en el $75.05 \%$ de los casos, la amplitud es mayor a 1.34 MW y que la mayor amplitud fue de 5.56 MW.

La Tabla 3, presenta un resumen de los modos inter-área con valores de amortiguamiento inferiores al $2.1 \%$ (menores al $3 \%$ existen 264 modos). En esta tabla, se aprecia que el "modo interárea" se presenta el día 18 de agosto del 2013, en horas de demanda baja y media, y son observables en la zona norte (Pomasqui, Santa Rosa y Totoras), por la presencia de oscilaciones entre el sistema ecuatoriano y el sistema colombiano, además, es observable también en Milagro, lo que sugiere una posible partición interna del SNI en dos áreas eléctricas.

\subsubsection{Modos locales}

En el período de estudio, se determina la presencia de 21 modos locales poco amortiguados, con una frecuencia de 0.93 a $1.85 \mathrm{~Hz}$, como muestra la Figura 9.

La Figura 10, presenta el histograma obtenido del análisis estadístico de las amplitudes que alcanzaron los modos locales poco amortiguados durante el período de análisis. De los resultados se observa que en el 95.23\% de los casos, la amplitud es mayor a 4.08 MW y que la mayor amplitud fue de 15.85 MW.

La Tabla 4, presenta un resumen de los modos locales con valores de amortiguamiento inferiores al $5 \%$ (no existieron modos con amortiguamiento menor al 3\%). En esta tabla, se aprecia que el "modo local" se presenta el día 17 de agosto de 2013, en horas de demanda media, pero están presentes también en demanda baja con amplitudes altas.

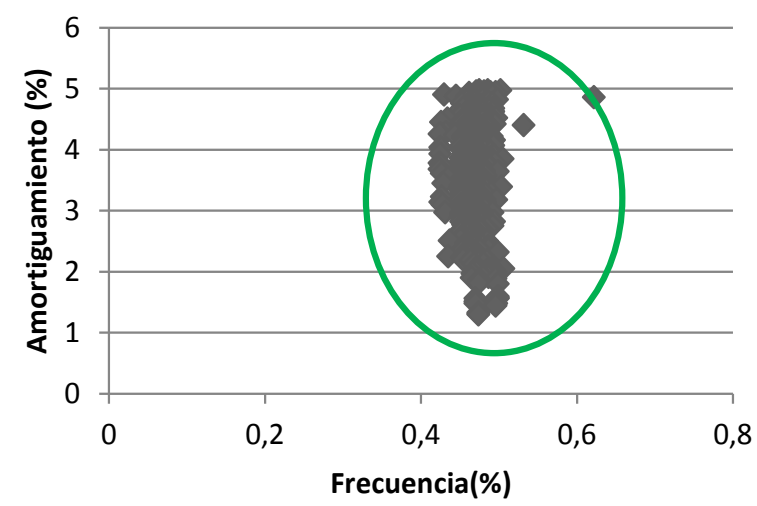

Figura 7. Modos inter-área con amortiguamiento inferior al $5 \%$

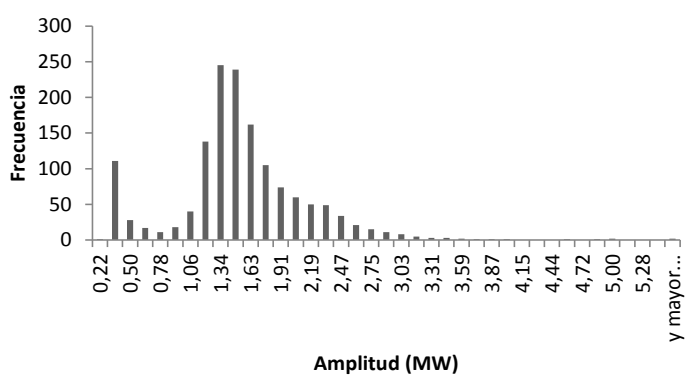

Figura 8. Histograma de amplitudes de los modos interárea poco amortiguados.

Tabla 3. Modos de control de "alta frecuencia" con amortiguamiento inferior al $3 \%$.

\begin{tabular}{lccccc}
\hline \multirow{2}{*}{ PMU } & & & \multicolumn{3}{c}{ Modo oscilatorio } \\
\cline { 4 - 6 } & Hora & Fecha & $\begin{array}{c}\text { Frec. } \\
\text { (Hz) }\end{array}$ & $\begin{array}{c}\text { Amp. } \\
\text { (MW) }\end{array}$ & $\begin{array}{c}\text { Amor. } \\
\text { (\%) }\end{array}$ \\
\hline TOTO_SROS1_230 & $6: 00$ & $18 / 08 / 2013$ & 0.496 & 2.3 & 1.98 \\
SROS_POMA1_230 & $8: 00$ & $18 / 08 / 2013$ & 0.485 & 4.8 & 1.98 \\
POMA_JAMO2_230 & $8: 00$ & $18 / 08 / 2013$ & 0.485 & 4.97 & 1.95 \\
SROS_TOTO2_230 & $6: 00$ & $18 / 08 / 2013$ & 0.496 & 2.19 & 1.94 \\
MILA_SIDE1_138 & $5: 00$ & $18 / 08 / 2013$ & 0.487 & 0.37 & 1.91 \\
SROS_TOTO1_230 & $6: 00$ & $18 / 08 / 2013$ & 0.496 & 2.2 & 1.89 \\
MILA_SIDE1_138 & $16: 00$ & $18 / 08 / 2013$ & 0.475 & 0.38 & 1.87 \\
SROS_TOTO1_230 & $9: 00$ & $18 / 08 / 2013$ & 0.499 & 2.19 & 1.59 \\
TOTO_SROS1_230 & $9: 00$ & $18 / 08 / 2013$ & 0.499 & 2.28 & 1.57 \\
POMA_JAMO3_230 & $7: 00$ & $18 / 08 / 2013$ & 0.47 & 5.51 & 1.51 \\
POMA_JAMO2_230 & $7: 00$ & $18 / 08 / 2013$ & 0.47 & 5.56 & 1.48 \\
POMA_JAMO3_230 & $9: 00$ & $18 / 08 / 2013$ & 0.496 & 2.53 & 1.44 \\
TOTO_SROS1_230 & $7: 00$ & $18 / 08 / 2013$ & 0.474 & 2.61 & 1.33 \\
SROS_TOTO2_230 & $7: 00$ & $18 / 08 / 2013$ & 0.474 & 2.49 & 1.31 \\
SROS_TOTO1_230 & $7: 00$ & $18 / 08 / 2013$ & 0.474 & 2.51 & 1.3 \\
\hline
\end{tabular}

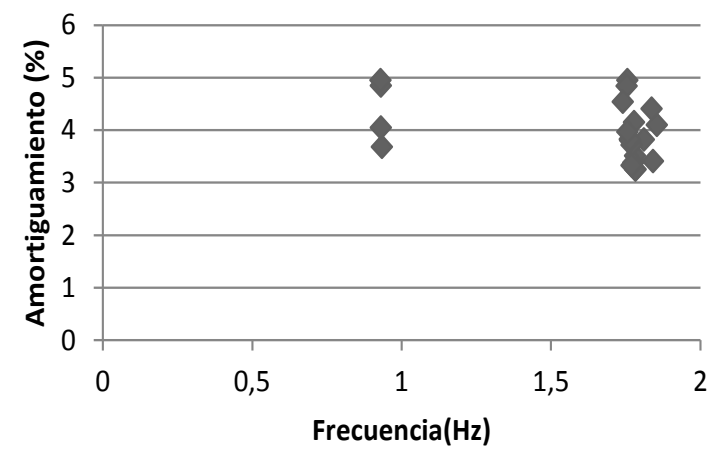

Figura 9. Modos locales detectados.

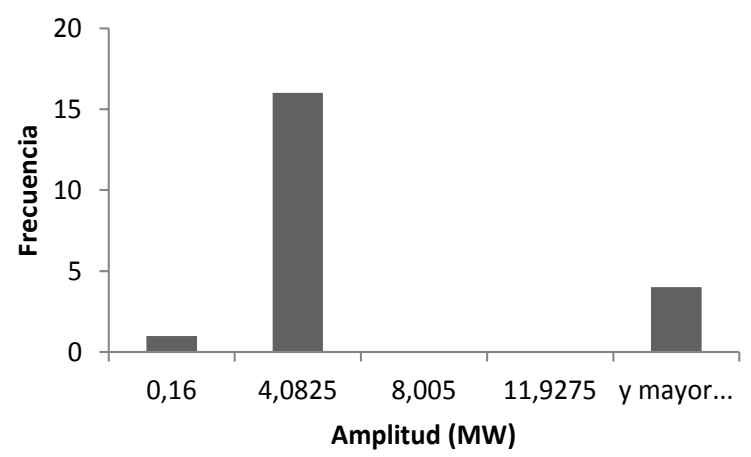

Figura 10. Histograma de amplitudes de los modos locales poco amortiguados. 
Tabla 4. Modos locales con amortiguamiento inferior al $5 \%$.

\begin{tabular}{|c|c|c|c|c|c|}
\hline \multirow[b]{2}{*}{ PMU } & \multirow[b]{2}{*}{ Hora } & \multirow[b]{2}{*}{ Fecha } & \multicolumn{3}{|c|}{ Modo oscilatorio } \\
\hline & & & $\begin{array}{l}\text { Frec. } \\
(\mathrm{Hz})\end{array}$ & $\begin{array}{c}\text { Amp. } \\
(\mathrm{MW})\end{array}$ & $\begin{array}{c}\text { Amor. } \\
(\%)\end{array}$ \\
\hline MILA_SIDE1_138 & $13: 00$ & $17 / 08 / 2013$ & 1.776 & 0.17 & 3.38 \\
\hline MILA_SIDE1_138 & $15: 00$ & $17 / 08 / 2013$ & 1.771 & 0.19 & 3.72 \\
\hline MILA_SIDE1_138 & $12: 00$ & $17 / 08 / 2013$ & 1.77 & 0.16 & 3.33 \\
\hline MOLI_AT1_138 & $13: 00$ & $17 / 08 / 2013$ & 1.766 & 0.93 & 3.81 \\
\hline ZHOR_MILA2_230 & $15: 00$ & $17 / 08 / 2013$ & 1.765 & 0.73 & 3.83 \\
\hline ZHOR_MILA2_230 & $12: 00$ & $17 / 08 / 2013$ & 1.756 & 0.6 & 4.95 \\
\hline MOLI_AT1_138 & $15: 00$ & $17 / 08 / 2013$ & 1.756 & 1.03 & 3.97 \\
\hline ZHOR_MILA $2 \_230$ & $14: 00$ & $17 / 08 / 2013$ & 1.754 & 0.72 & 4.84 \\
\hline MOLI_AT1_138 & $14: 00$ & $17 / 08 / 2013$ & 1.741 & 1.05 & 4.54 \\
\hline MOLI_TOTO_230 & 7:00 & $17 / 08 / 2013$ & 0.935 & 15.85 & 3.68 \\
\hline SROS_TOTO2_230 & $6: 00$ & $17 / 08 / 2013$ & 0.931 & 14.72 & 4.85 \\
\hline TOTO_SROS1_230 & $7: 00$ & $17 / 08 / 2013$ & 0.931 & 15.76 & 4.05 \\
\hline SROS TOTO1 230 & $6: 00$ & $17 / 08 / 2013$ & 0.93 & 14.78 & 4.95 \\
\hline
\end{tabular}

\subsubsection{Análisis de resultados}

En el período de análisis, se ha determinado que en el SNI existen cuatro tipos de modos oscilatorios poco amortiguados: de control de muy baja frecuencia, de control de alta frecuencia, interárea y locales. Los modos oscilatorios son dominantes en determinados períodos del día, de la siguiente forma:

Modo de control de muy baja frecuencia: períodos de demanda media.

Modos de control de alta frecuencia: períodos de demanda media.

Modos interárea: períodos de demanda baja y media.

Modos locales: períodos de demanda media.

El día 17 de agosto, por trabajos programados de CELEC EP - TRANSELECRIC, se abre a las 05:55 el autotransformador ATT 230/138 kV de la S/E Totoras. Esta apertura, ocasiona oscilaciones locales poco amortiguadas y con amplitudes altas, de hasta 15.85 MW en promedio, como se observa en la Figura 11. El día 18 de agosto no se programa importación de energía desde el sistema colombiano, pero se exportó 1792 MWh hacia el sistema colombiano por el enlace de $230 \mathrm{kV}$ y Paute operó con nueve máquinas disponibles. Este día se observa que durante el período que se exporta a Colombia, la excitación del modo interárea presenta oscilaciones sostenidas con amortiguamientos menores al $3 \%$ y amplitudes de hasta 5.56 MW. Adicionalmente, el estado operativo del SNI fue $76 \%$ de generación hidráulica, 24\% generación térmica. La Figura 12, presenta el evento oscilatorio interárea descrito.

\section{Estrategias utilizadas para la implementación del sistema WAMS en el SNI}

La investigación realizada, tanto del estado del arte de la tecnología WAMS como de las particularidades

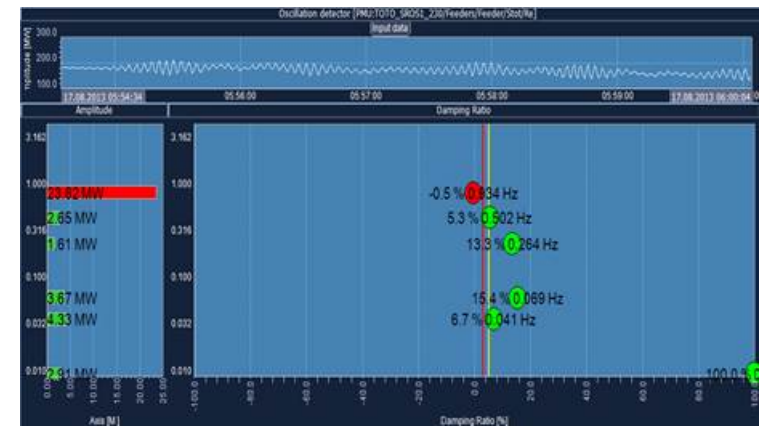

Figura 11. Modos Locales PMU Totoras-Santa Rosa, para el 17-08-2013. Fuente: CENACE, Aplicación WAProtector.

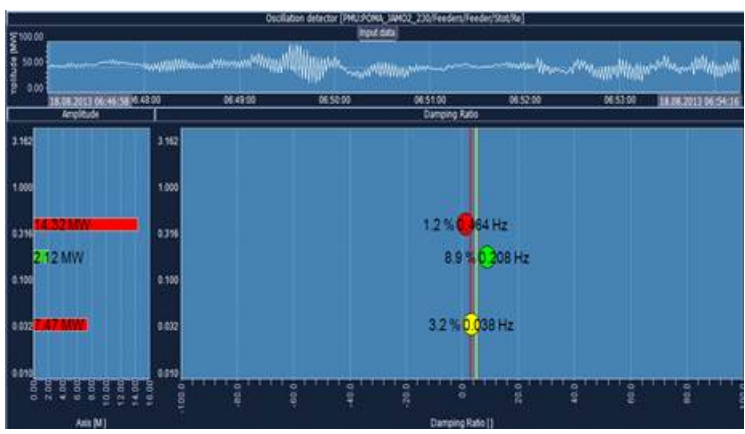

Figura 12. Modos Inter-área PMU: Pomasqui-Jamondino 2, para el 18-08-2013.. Fuente: CENACE, Aplicación WAProtector.

en la operación del sistema eléctrico de Ecuador, permite establecer ciertos criterios que podrían considerarse para la implementación de un sistema WAMS generalizado:

- Fomentar capacitación especializada de los profesionales encargados del diseño, implementación y operación del sistema WAMS. Esta capacitación deberá incluir el seguimiento de las mejores prácticas y experiencias.

- Formar un grupo de trabajo interdisciplinario de profesionales que lidere las actividades de implementación del sistema WAMS.

- En vista de que se trata de una tecnología relativamente nueva, es necesario realizar pruebas de concepto o proyectos piloto con diferentes suministradores de aplicaciones WAMS y PMU, con el fin de evaluar sus principales características y minimizar riesgos técnicos y operacionales.

- Adoptar al inicio un criterio de ubicación de PMU coherente con la realizada de cada sistema, para lo cual se pueden aplicar las metodologías descritas en el numeral 3 anterior. Como criterio inicial se debería empezar con la ubicación considerando la supervisión angular del SEP dando 
prioridad a las barras del anillo troncal del sistema de transmisión, en las subestaciones frontera de interconexiones eléctricas y en las barras de los grandes centros de carga.

- Definir la velocidad de muestreo para la adquisición de información de las PMU, tomando en cuenta el medio y ancho de banda del sistema de comunicaciones.

- Determinar las variables eléctricas a extraerse directamente de las PMU y aquellas que serán calculadas por el PDC.

- Definir la arquitectura del sistema: PDC central, local o regional.

- Definir la capacidad física de almacenamiento del sistema en función del intervalo de muestreo de las variables.

- Asegurarse que las señales de voltajes y corrientes de entrada a los PMU sean de alta calidad.

- Definir un sistema de comunicaciones adecuado y dedicado que garantice la transmisión de datos y su confiabilidad.

- Para la implementación definitiva, realizar un concurso convocando a empresas de prestigio mundial en base a requerimientos funcionales apropiados.

- En el ámbito del operador del sistema eléctrico, desarrollar nuevos procesos/subprocesos de planificación y operación del SNI que hagan uso de la información que aporta el sistema WAMS.

- Generar procedimientos para la operación y mantenimiento de la aplicación WAMS, a más de procurar con el proveedor los acuerdos de garantía técnica y actualizaciones del sistema conforme existan nuevas versiones o surjan nuevas necesidades del usuario.

\section{Conclusiones}

La implementación de WAMS provee información de alta precisión y resolución para la planificación, operación y análisis posoperativo del SNI. Por tal motivo, es necesario complementar el enfoque del estudio del SEP para incluir su caracterización en función de la diferencia angular de los fasores de voltaje y de los parámetros de estabilidad oscilatoria, como amplitud y amortiguamiento de los modos oscilatorios.

Con la implementación del sistema WAMS y los análisis posoperativos realizados, ha sido posible identificar el comportamiento dinámico del sistema, determinar zonas coherentes de influencia oscilatoria, máquinas que participan en las oscilaciones, estados operativos que influyen los modos de oscilación y ciertos comportamientos dinámicos que antes no eran visibles con el tradicional sistema SCADA/EMS.

Con el empleo del sistema WAMS se marca el inicio del proceso de caracterización del sistema de potencia, lo que permitirá definir los límites reales de alertas y alarmas para los diferentes módulos de detección. Estos análisis conducirán a la determinación de recomendaciones operativas sobre cómo actuar al presentarse ciertos eventos en el SNI, con el objetivo de mejorar considerablemente los criterios y forma de operar, incrementándose la seguridad y confiabilidad del SEP.

De los análisis realizados semanalmente desde mayo del 2013, se ha observado que ciertos estados operativos y topológicos, excitan determinados modos oscilatorios, como por ejemplo la exportación a Colombia, los porcentajes de generación hidroeléctrica y térmica, la operación de ciertas máquinas, la apertura de ciertos elementos del SNI, entre otros. Análisis más especializados (como el multivariante) permitirán identificar estados operativos potencialmente peligrosos y sugerir recomendaciones para la planificación y operación del SNI. Futuras investigaciones son necesarias para llegar a término este objetivo.

\section{Referencias}

[1] Arbiter Systems, Inc., Manual de Usuario 1133A. [Online]. Available: http://www.arbiter.com/catalog/product/ model-1133a-power-sentinel.php

[2] R. Cimadevilla, "Fundamentos de la medición de sincrofasores," in XIII ERIAC - Décimo tercer encuentro regional iberoamericano de Cigré, Puerto Iguazú, 2009, p. 10.

[3] C. Martínez, M. Parashar, J. Dyer, and J. Coroas, "Phasor data requirements for real time wide-area monitoring, control and protection applications," EIPP White Paper, p. 8, 2005.

[4] A. G. Phadke and J. S. Thorp, Synchronized phasor measurements and their applications. Springer, 2008.

[5] K. Martin, G. Benmouyal, M. Adamiak, M. Begovic, J. Burnett, R.O., K. Carr, A. Cobb, J. Kusters, S. Horowitz, G. Jensen, G. Michel, R. Murphy, A. Phadke, M. Sachdev, and J. Thorp, IEEE Standard for Synchrophasors for Power Systems, Std. 1, 1998.

[6] M. Campos and M. Arias, "Ubicación óptima de unidades de medición fasorial aplicando swarm 
intelligence," in IEEE/PES 2010 Transmition \& Distribution Conference and Exposition Latin America, Brazil, 2010, p. 8. [Online]. Available: http: / /abplan.ufsc.br/congressos/Induscon\% 202010/fscommand/web/docs/T1100.pdf

[7] Elpros, Manual de WAProtector, 2013.

[8] P. Kundur, Power system stability and control. McGraw-Hill, Inc., 2001, vol. 12.
[9] C. Juárez and D. Colomé, "Tendencias en la supervisión en tiempo real de la estabilidad de pequeña señal de sistemas de potencia," 2009, pp. 1-8.

[10] S. P. Teeuwsen, "Oscillatory stability assessment of power systems using computational intelligence," Ph.D. dissertation, Universitätsbibliothek Duisburg, 2005. 